\title{
BOEHMIANS ON MANIFOLDS
}

\author{
PIOTR MIKUSIŃSKI
}

(Received 13 March 2000)

\begin{abstract}
The construction of Boehmians on a manifold requires a commutative convolution structure. We present such constructions in two specific cases: an $\mathrm{N}$-dimensional torus and an $\mathrm{N}$-dimensional sphere. Then we formulate conditions under which a construction of Boehmians on a manifold is possible.
\end{abstract}

Keywords and phrases. Boehmians, manifold, convolution, locally compact groups.

2000 Mathematics Subject Classification. Primary 44A40, 43A85; Secondary 44A35, 46F99.

1. Introduction. Boehmians were first constructed as a generalization of regular Mikusiński operators [1, 3]. The purpose of that construction was to produce a space of generalized functions defined as convolution quotients, but without any restriction on the support. Almost all papers on Boehmians published to date concern objects defined on $\mathbb{R}^{N}$. Because of a certain inflexibility of Boehmians (see [6]) and their global character, there is no obvious way to construct Boehmians on manifolds. In this paper, we present a framework that seems to be well suited for Boehmians on manifolds. Instead of defining Boehmians locally on a manifold by coordinate patches, we use the "internal convolution structure" of a manifold. To this aim we choose the language of locally compact groups, which provides a very natural setting for the construction. Boehmians on locally compact Abelian groups were already considered by Nemzer in [9]. However, many manifolds do not have a natural commutative group structure. The method proposed here generalizes the idea used in [7] where a construction of Boehmians on the $N$-dimensional sphere uses the convolution algebra of continuous functions defined on the group of rotations of the sphere. A construction of Boehmians on the sphere was given earlier in [8]. The method used there is more dependent on special properties of the sphere and does not seem to lead to natural generalizations.

The minimal structure necessary for the construction of Boehmians consists of the following elements:

(I) a nonempty set $\mathscr{X}$,

(II) a commutative semigroup $(\mathscr{Y}, *)$,

(III) an operation $\odot: \mathscr{X} \times \mathscr{Y} \rightarrow \mathscr{X}$ such that for every $x \in \mathscr{X}$ and $s_{1}, s_{2} \in \mathscr{Y}$ we have $x \odot\left(s_{1} * s_{2}\right)=\left(x \odot s_{1}\right) \odot s_{2}$,

(IV) a nonempty collection $\Delta \subset \mathscr{G}^{\mathbb{N}}$ such that

(a) if $x, y \in \mathscr{X},\left(s_{n}\right) \in \Delta$, and $x \odot s_{n}=y \odot s_{n}$ for all $n \in \mathbb{N}$, then $x=y$,

(b) if $\left(s_{n}\right),\left(t_{n}\right) \in \Delta$, then $\left(s_{n} * t_{n}\right) \in \Delta$.

Elements of $\Delta$ will be called delta sequences. Let

$$
\mathscr{A}=\left\{\left(x_{n}, s_{n}\right): x_{n} \in \mathscr{X},\left(s_{n}\right) \in \Delta \text {, and } x_{n} \odot s_{m}=x_{m} \odot s_{n} \forall m, n \in \mathbb{N}\right\} \text {. }
$$


If $\left(x_{n}, s_{n}\right),\left(y_{n}, t_{n}\right) \in \mathscr{A}$ and $x_{n} \odot t_{m}=y_{m} \odot s_{n}$, for all $m, n \in \mathbb{N}$, then we write $\left(x_{n}, s_{n}\right) \sim$ $\left(y_{n}, t_{n}\right)$. The relation $\sim$ is an equivalence in $\mathscr{A}$. It is trivially reflexive and symmetric. To prove that it is transitive assume that $\left(x_{n}, r_{n}\right) \sim\left(y_{n}, s_{n}\right)$ and $\left(y_{n}, s_{n}\right) \sim\left(z_{n}, t_{n}\right)$. Fix an arbitrary $k \in \mathbb{N}$. Then

$$
\begin{aligned}
\left(x_{n} \odot t_{m}\right) \odot s_{k} & =x_{n} \odot\left(t_{m} * s_{k}\right)=x_{n} \odot\left(s_{k} * t_{m}\right)=\left(x_{n} \odot s_{k}\right) \odot t_{m} \\
& =\left(y_{k} \odot r_{n}\right) \odot t_{m}=\left(y_{k} \odot t_{m}\right) \odot r_{n}=\left(z_{m} \odot s_{k}\right) \odot r_{n} \\
& =\left(z_{m} \odot r_{n}\right) \odot s_{k} .
\end{aligned}
$$

Since $m, n \in \mathbb{N}$ are arbitrary and $\left(x_{n} \odot t_{m}\right) \odot s_{k}=\left(z_{m} \odot r_{n}\right) \odot s_{k}$ for all $k \in \mathbb{N}$, we must have $x_{n} \odot t_{m}=z_{m} \odot r_{n}$ for all $m, n \in \mathbb{N}$.

The space of equivalence classes in $\mathscr{A}$ will be denoted by $\mathscr{B}(\mathscr{X})$. Elements of $\mathscr{B}(\mathscr{X})$ are called Boehmians. To simplify the notation, the equivalence class of $\left(x_{n}, s_{n}\right)$ will be denoted by $x_{n} / s_{n}$. There is a canonical embedding of $\mathscr{X}$ into $\mathscr{B}(\mathscr{X})$ :

$$
x \longmapsto \frac{x \odot s_{n}}{s_{n}} .
$$

It is easy to check that this mapping is independent of a particular delta sequence $\left(s_{n}\right)$. The operation $\odot$ can be extended to $\mathscr{B}(\mathscr{X}) \times \mathscr{Y}$ :

$$
\frac{x_{n}}{s_{n}} \odot t=\frac{x_{n} \odot t}{s_{n}} .
$$

Conditions (III) and (IV)(a) hold for this extension. If $\mathscr{X}$ has an additional structure, it can usually be extended to $\mathscr{B}(\mathscr{X})$ (cf. [4] or [5]).

In most examples we have $\mathscr{S} \subset \mathscr{X}$ and $\odot=*$. For applications to generalized functions we usually take $\mathscr{X}$ and $\mathscr{Y}$ to be spaces of functions defined on a common domain and the operation is a convolution product.

2. Boehmians on $T^{N}$ and $S^{N-1}$. First we consider Boehmians on the $N$-dimensional torus. Let $T=[0,1]$ and let + indicate addition modulo 1 . This operation extends to $T^{N}$ in an obvious way and makes $T^{N}$ a commutative group. For $f, g \in L^{1}\left(T^{N}\right)$, we define

$$
(f * g)(x)=\int_{T^{N}} f(z) g(x-z) d z
$$

where $d z$ indicates integration with respect to the Lebesgue measure on $T^{N}$. Let $\mathscr{X}=$ $\mathscr{S}=L^{1}\left(T^{N}\right), \odot=*$ as defined in (2.1), and let $\Delta$ be the collection of all sequences $\varphi_{1}$, $\varphi_{2}, \ldots \in L^{1}\left(T^{N}\right)$ satisfying the following conditions:

(1) $\varphi_{n} \geq 0$ for all $n \in \mathbb{N}$,

(2) $\int_{T^{N}} \varphi_{n}=1$ for all $n \in \mathbb{N}$,

(3) for every $\varepsilon \in(0,1 / 2)$ there exists an $n_{0} \in \mathbb{N}$ such that $\varphi_{n}(x)=0$ for all $x \in$ $[\varepsilon, 1-\varepsilon]^{N}$ and for all $n \geq n_{0}$.

Clearly, conditions (I), (II), (III), and (IV) are satisfied and thus the construction of Boehmians on $T^{N}$ is possible. Note that we do not encounter any difficulties in this example since $T^{N}$ and, consequently, the convolution algebra $L^{1}\left(T^{N}\right)$ are commutative. 
Now we turn to the sphere $S^{N-1}$. There is no natural group structure on $S^{N-1}$ and thus no natural convolution in $L^{1}\left(S^{N-1}\right)$.

Let $e_{N}=(0, \ldots, 0,1) \in \mathbb{R}^{N}$ and let $\mathscr{T}$ denote the set of all rotations in $\mathbb{R}^{n}$. Let $\mathscr{T}_{0}=$ $\left\{T \in \mathcal{T} \mid T e_{N}=e_{N}\right\}$ and

$$
\mathscr{P}=\left\{\varphi \in L^{\infty}\left(S^{N-1}\right) \mid \varphi \circ T=\varphi \forall T \in \mathscr{T}_{0}\right\}
$$

The convolution of $f \in L^{1}\left(S^{N-1}\right)$ and $\varphi \in \mathscr{P}$ is defined by

$$
(f * \varphi)(x)=(f * \varphi)\left(T_{x} e_{N}\right)=\int_{S^{N-1}} f(z) \varphi\left(T_{x}^{-1} z\right) d z,
$$

where $x \in S^{N-1}$, and $T_{x} \in \mathscr{T}$ is such that $T_{x} e_{N}=x$. Since $\varphi \in \mathscr{P}, f * \varphi$ does not depend on the choice of a particular $T_{x} \in \mathcal{T}$, and thus $f * \varphi$ is well defined. Now we can take $\mathscr{X}=L^{1}\left(S^{N-1}\right), \mathscr{S}=\mathscr{P}, *$ and $\odot$ both defined by (2.3), and $\Delta$ to be the collection of all sequences $\varphi_{1}, \varphi_{2}, \ldots \in \mathscr{P}$ satisfying the following conditions:

(1) $\varphi_{n} \geq 0$ for all $n \in \mathbb{N}$,

(2) $\int_{S^{N-1}} \varphi_{n}=1$ for all $n \in \mathbb{N}$,

(3) for every neighborhood $V$ of $e_{N}$ there exists an $n_{0} \in \mathbb{N}$ such that $\operatorname{supp} \varphi_{n} \subset V$ for all $n \geq n_{0}$.

One can prove that conditions (I), (II), (III), and (IV) are satisfied and the construction of Boehmians on $S^{N-1}$ is possible. This construction of Boehmians on the sphere $S^{N-1}$ in $\mathbb{R}^{N}$ was presented in [8].

3. Boehmians on locally compact groups. The framework of locally compact groups is particularly well suited for the construction of Boehmians. It provides a simple way of constructing Boehmians on certain manifolds that are locally compact groups. Moreover, some results from this section will be useful in the more general situation considered in Section 4.

Let $G$ be a locally compact group, $e$ the identity element of $G$, and $L^{1}(G)$ the convolution algebra of integrable functions with respect to the left Haar measure on $G$. The convolution in $L^{1}(G)$ is defined by

$$
(\varphi * \psi)(x)=\int_{G} \varphi(z) \psi\left(z^{-1} x\right) d x .
$$

By $\mathscr{L}(G)$ we denote the center of $L^{1}(G)$, i.e., $\varphi \in \mathscr{L}(G)$ if and only if $f * \varphi=\varphi * f$ for all $f \in L^{1}(G)$. The following simple characterization of $\mathscr{L}(G)$ will be useful (see [2]).

LEMMA 3.1. Let $G$ be a unimodular locally compact group. Then $f \in \mathscr{L}(G)$ if and only if $f(x y)=f(y x)$ for all $x, y \in G$.

A sequence $\varphi_{1}, \varphi_{2}, \ldots \in L^{1}(G)$, will be called a delta sequence if

(1) $\varphi_{n} \in \mathscr{L}(G)$ for all $n \in \mathbb{N}$,

(2) $\varphi_{n} \geq 0$ for all $n \in \mathbb{N}$,

(3) $\int_{G} \varphi_{n}=1$ for all $n \in \mathbb{N}$,

(4) for every neighborhood $V$ of $e$ there exists an $n_{0} \in \mathbb{N}$ such that $\operatorname{supp} \varphi_{n} \subset V$ for all $n \geq n_{0}$. 
LEMMA 3.2. Let $\left(\varphi_{n}\right)$ and $\left(\psi_{n}\right)$ be delta sequences.

(a) If $f \in L^{1}(G)$ and $f * \varphi_{n}=0$ for all $n \in \mathbb{N}$, then $f=0$.

(b) $\left(\varphi_{n} * \psi_{n}\right)$ is a delta sequence.

DefinITION 3.3. A locally compact group $G$ is called a $B$-group if there exists a delta sequence in $L^{1}(G)$.

In every locally compact group there exist approximate identities. Since we need existence of delta sequences, as opposed to nets, we will have to assume that $G$ is first countable. Clearly every first countable locally compact Abelian group is a B-group.

THEOREM 3.4. Every first countable compact group is a B-group.

Proof. It suffices to show that for every neighborhood $U$ of $e$ there is a $\varphi \in \mathscr{L}(G)$ such that supp $\varphi \subset U$. Let $U$ be an arbitrary neighborhood of $e$. Define

$$
F=\left\{z x z^{-1}: z \in G \text { and } x \in G \backslash U\right\} \quad \text { and } \quad V=G \backslash F .
$$

Then $V$ is a neighborhood of $e$. Clearly, $e \in V$. Moreover, since $F$ is the continuous image of the compact set $G \times(G \backslash U)$, it is a closed subset of $G$. Thus $V$ is open. Let $\psi$ be the characteristic function of $V$. Define

$$
\varphi(x)=\int \psi\left(z x z^{-1}\right) d z .
$$

Since $\psi\left(z x z^{-1}\right)=0$ for every $z \in G$ and $x \in G \backslash U, \varphi(x)=0$ for every $x \in G \backslash U$, which means that $\operatorname{supp} \varphi \subset U$. Finally, to see that $\varphi \in \mathscr{L}(G)$, note that $\varphi(x y)=\varphi(y x)$ for all $x, y \in G$ and then use Lemma 3.1.

If $G$ is a B-group, then we can take $\mathscr{L}=L^{1}(G), S=\mathscr{L}(G)$, the convolution in $L^{1}(G)$ for both $\odot$ and $*$, and finally, the collection of all delta sequences in $\mathscr{L}(G)$ for $\Delta$. Then all conditions necessary for the construction of Boehmians are satisfied. The obtained space of Boehmians will be denoted by $\mathscr{B}\left(L^{1}(G)\right)$, or simply by $\mathscr{B}(G)$.

As in the general case, $L^{1}(G)$ can be identified with a subset of $\mathscr{B}(G)$ via

$$
\iota(f)=\frac{f * \varphi_{n}}{\varphi_{n}} .
$$

Then $\iota(f * \psi)=\iota(f) * \psi$. If, for some $F \in \mathscr{B}(G), f \in L^{1}(G)$, and $\varphi \in \mathscr{L}(G)$, we have $F * \varphi=\iota(f)$, we simply write $F * \varphi=f$. For example, if $F=f_{n} / \varphi_{n}$, then $F * \varphi_{k}=f_{k}$ for any $k \in \mathbb{N}$.

A sequence of Boehmians $\left(F_{n}\right)$ is said to converge to zero in $\mathscr{B}(G)$ if there exists a delta sequence $\left(\varphi_{n}\right)$ such that $F_{n} * \varphi_{n} \in L^{1}(G)$, for all $n \in \mathbb{N}$, and $F_{n} * \varphi_{n} \rightarrow 0$ in $L^{1}(G)$. Convergence to an arbitrary limit $F$ is defined by $F_{n}-F \rightarrow 0$ in $\mathscr{B}(G)$. With this convergence in $\mathscr{B}(G)$ the mapping $\iota$ is continuous.

4. Boehmians on manifolds. The method presented in this section was suggested by the construction of Boehmians on the sphere introduced in [7]. It is different from the approach used in [8]. Our intention here is to describe a method that can be applied to a variety of manifolds. 
Let $\mathcal{M}$ be a $\sigma$-compact manifold and let $\mathscr{T}$ be a locally compact group of transformations on $\mu$ such that:

(a) every $T \in \mathcal{T}$ is a homeomorphism of $\mathcal{M}$,

(b) for every $f \in \mathscr{C}(\mathcal{M})$ the mapping $T \mapsto f \circ T$ is continuous,

(c) for every $x, y \in \mathcal{M}$ there exists a $T \in \mathcal{T}$ such that $T x=y$.

For $f \in \mathscr{C}(\mathcal{M})$ and $\varphi \in L^{1}(\mathscr{T})$ define

$$
(f \odot \varphi)(x)=\int_{\mathscr{T}} f\left(T^{-1} x\right) \varphi(T) d T .
$$

LEMMA 4.1. Let $\left(\varphi_{n}\right)$ be a delta sequence in $L^{1}(J)$ and let $f \in \mathscr{C}(\mathcal{M})$. Then $f \odot \varphi_{n} \rightarrow$ $f$ in $\mathscr{C}(M)$.

Proof. Let $K$ be a compact subset of $\mathcal{M}$. Then

$$
\begin{aligned}
\sup _{x \in K}\left|f \odot \varphi_{n}(x)-f(x)\right| & =\sup _{x \in K}\left|\int_{\mathscr{T}} f\left(T^{-1} x\right) \varphi_{n}(T) d T-f(x)\right| \\
& =\sup _{x \in K}\left|\int_{\mathscr{T}} f\left(T^{-1} x\right) \varphi_{n}(T) d T-f(x) \int_{\mathscr{T}} \varphi_{n}(T) d T\right| \\
& \leq \sup _{x \in K} \int_{\mathscr{T}}\left|\left(f\left(T^{-1} x\right)-f(x)\right) \varphi_{n}(T)\right| d T \\
& \leq \sup _{x \in K} \sup _{T \in \operatorname{supp} \varphi_{n}}\left|f\left(T^{-1} x\right)-f(x)\right| .
\end{aligned}
$$

Since supp $\varphi_{n} \rightarrow I$ ( $I$ denotes the identity operator on $M$ ), we have

$$
\sup _{x \in K} \sup _{T \in \operatorname{supp} \varphi_{n}}\left|f\left(T^{-1} x\right)-f(x)\right| \longrightarrow 0 \text { as } n \rightarrow \infty,
$$

by (b).

LEMMA 4.2. For $f \in \mathscr{C}(\mathcal{M})$ and $\varphi, \psi \in L^{1}(J)$ we have

$$
f \odot(\varphi * \psi)=(f \odot \psi) \odot \varphi .
$$

ProOF.

$$
\begin{aligned}
(f \odot(\varphi * \psi))(x) & =\int_{\mathscr{T}} f\left(T^{-1} x\right)(\varphi * \psi)(T) d T \\
& =\int_{\mathscr{T}} f\left(T^{-1} x\right) \int_{\mathscr{T}} \varphi(S) \psi\left(S^{-1} T\right) d S d T \\
& =\int_{\mathscr{T}} \int_{\mathscr{T}} f\left(T^{-1} x\right) \psi\left(S^{-1} T\right) d T \varphi(S) d S \\
& =\int_{\mathscr{T}} \int_{\mathscr{T}} f\left(T^{-1} S^{-1} x\right) \psi(T) d T \varphi(S) d S \\
& =\int_{\mathscr{T}}(f \odot \psi)\left(S^{-1} x\right) \varphi(S) d S \\
& =((f \odot \psi) \odot \varphi)(x) .
\end{aligned}
$$

Note that for $\varphi, \psi \in \mathscr{L}(J)$ we have

$$
f \odot(\varphi * \psi)=(f \odot \varphi) \odot \psi \quad \text { for every } f \in \mathscr{C}(\mathcal{M}) .
$$


If $\mathscr{T}$ is a $B$-group, then the construction of Boehmians is possible for $\mathscr{X}=\mathscr{C}(\mathcal{M})$, $\mathscr{S}=\mathscr{L}(\mathscr{T})$, and $*$ and $\odot$ as defined above. For example, if we can find a locally compact group of transformations $\mathcal{T}$ on $\mathcal{M}$ that is first countable and Abelian or first countable and compact, then we can construct Boehmians on $\mathcal{M}$. For $\mathcal{M}=S^{N-1}$ we can use the group of all rotations. It is a first countable compact group and therefore the described method can be used. Note that this approach is not a direct generalization of the construction sketched in Section 2.

\section{REFERENCES}

[1] T. K. Boehme, The support of Mikusiński operators, Trans. Amer. Math. Soc. 176 (1973), 319-334. MR 47\#2281. Zbl 268.44005.

[2] L. H. Loomis, An Introduction to Abstract Harmonic Analysis, D. Van Nostrand Company, Inc., Toronto, New York, London, 1953. MR 14,883c. Zbl 052.11701.

[3] J. Mikusiński and P. Mikusiński, Quotients de suites et leurs applications dans l'analyse fonctionnelle [Quotients of sequences and their applications in functional analysis], C. R. Acad. Sci. Paris Sér. I Math. 293 (1981), no. 9, 463-464 (French). MR 83a:46048. Zbl 495.44006 .

[4] P. Mikusiński, Convergence of Boehmians, Japan. J. Math. (N.S.) 9 (1983), no. 1, 159-179. MR 85h:46063. Zbl 524.44005.

[5] _ Boehmians and generalized functions, Acta Math. Hungar. 51 (1988), no. 3-4, 271281. MR 90a:46090. Zbl 652.44005.

[6] _ On flexibility of Boehmians, Integral Transform. Spec. Funct. 4 (1996), no. 1-2, 141-146, Proceedings of the Conference "Different Aspects of Differentiability", II (Warsaw, 1995). MR 99e:44004. Zbl 863.44004.

[7] P. Mikusiński and M. Morimoto, Boehmians on the sphere and there spherical harmonic expansions, Fract. Calc. Appl. Anal., to appear.

[8] P. Mikusiński and B. A. Pyle, Boehmians on the sphere, Integral Transform. Spec. Funct. 10 (2000), 93-100.

[9] D. Nemzer, The Boehmians as an F-space, Ph.D. thesis, University of California, Santa Barbara, 1984.

Piotr Mikusiński: Department of Mathematics, University of Central Florida, ORLANDO, FL 32816-1364, USA

E-mail address: piotrm@mai 1 . ucf.edu 


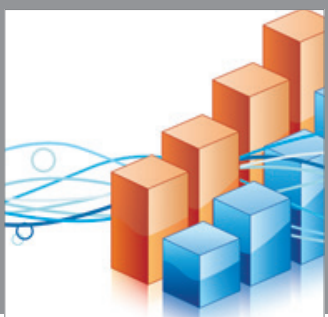

Advances in

Operations Research

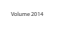

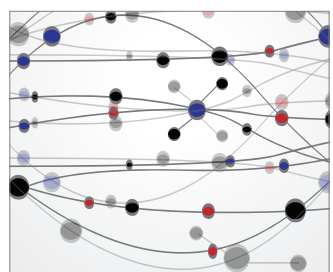

\section{The Scientific} World Journal
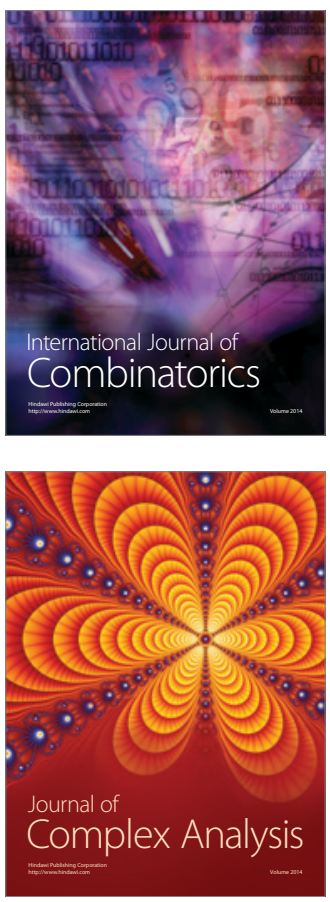

International Journal of

Mathematics and

Mathematical

Sciences
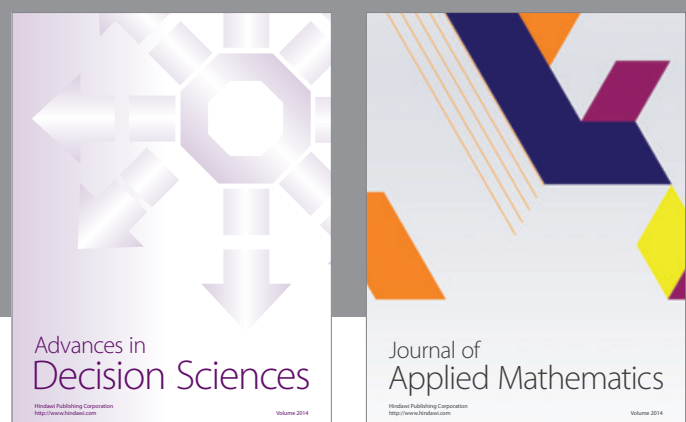

Journal of

Applied Mathematics
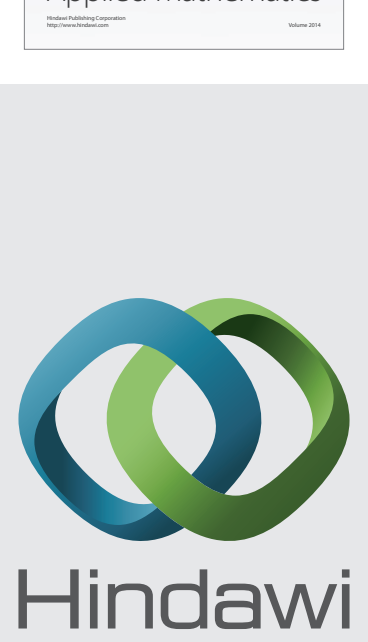

Submit your manuscripts at http://www.hindawi.com
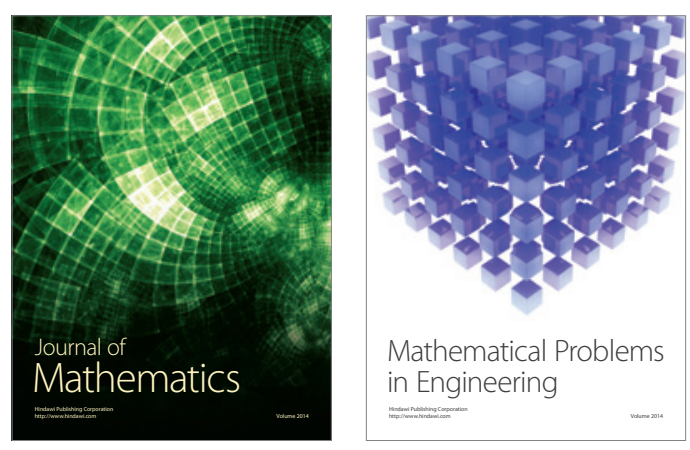

Mathematical Problems in Engineering
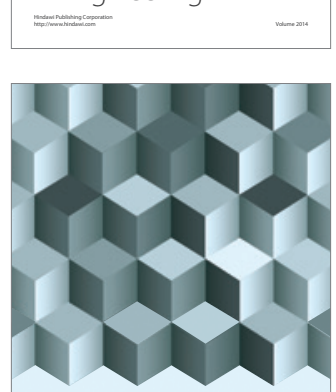

Journal of

Function Spaces
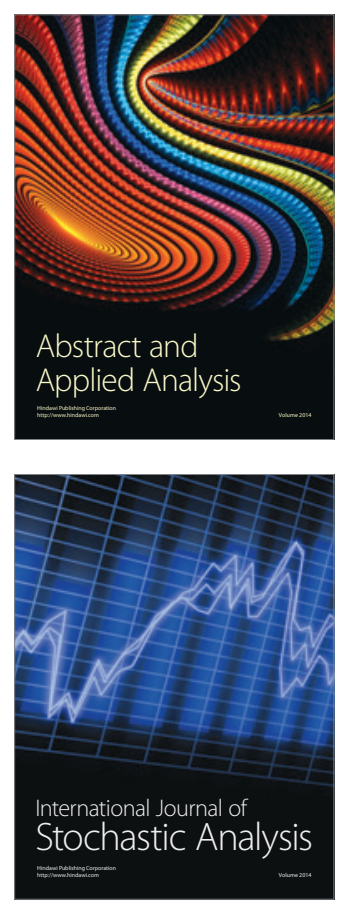

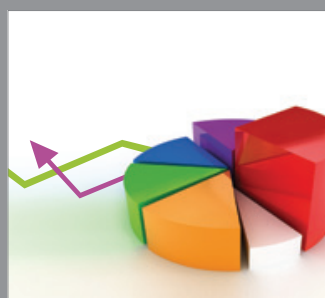

ournal of

Probability and Statistics

Promensencen
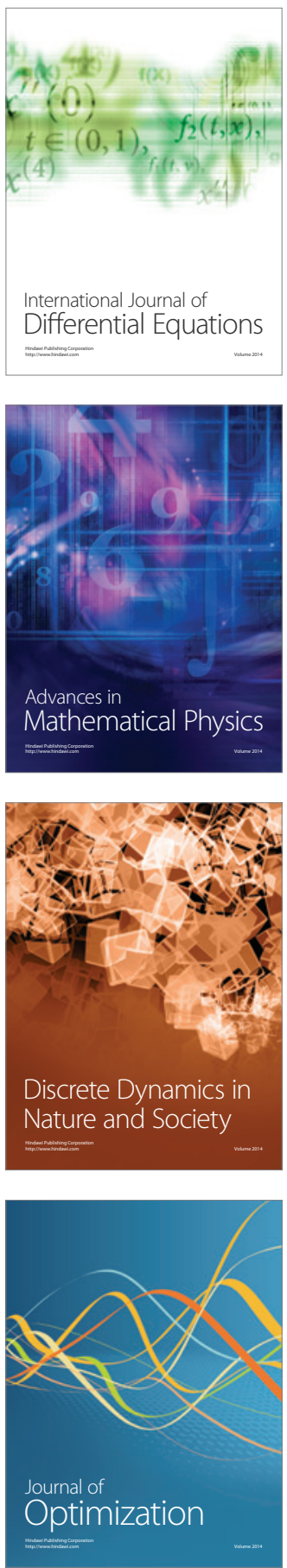